\title{
Revisión
}

\section{Modelo ideal de nefroprevención para Colombia}

\author{
Juan Zakzuk Perez ${ }^{\text {(D) }}{ }^{\square}$, Josber Vargas Torres ${ }^{\text {(D) }}{ }^{2}$, María Urzola Suárez ${ }^{(\mathbb{D} 2}$, \\ María Piñerez Ochoa ${ }^{\mathbb{D}}{ }^{1}$, German Arenas Hoyos ${ }^{(D)}{ }^{1}$, y Oscar Ocampo \\ Henao (D)1
}

${ }^{1}$ Universidad de Cartagena, Facultad de Medicina, Cartagena de Indias, Colombia.

${ }^{2}$ Universidad del Sinú, Seccional Cartagena, Facultad de Ciencias de la Salud, Cartagena de Indias, Colombia.

Cómo citar: Pérez JZ, Vargas Torres J, Suárez MU, Piñerez Ochoa M, Arenas Hoyos G, Ocampo Henao O. Modelo ideal de nefroprevención para Colombia. Rev. Colomb. Nefrol. 2021 8(3), e431. https://doi.org/10.22265/acnef.8.3.431

Recibido:

03/Mar/2020

Aceptado:

17/Jun/2020

Publicado:

18/Ago/2021

\section{Resumen}

La enfermedad renal crónica (ERC) es una patología altamente prevalente en países en vía de desarrollo como Colombia, en donde afecta a gran parte de la población. Esta es una enfermedad que afecta la calidad de vida y la longevidad de los pacientes, así como los costos del sistema de salud.

La ERC tiene un carácter progresivo e irreversible, por lo que intervenirla en etapas tempranas resulta ser una medida altamente costo-efectiva; a partir de esto surge el concepto de nefroprevención, mediante la cual, teniendo en cuenta los niveles de prevención primaria, secundaria y terciaria, se puede lograr una identificación precoz y la interrupción o enlentecimiento de la progresión, y además se puede implementar un manejo oportuno e integral del daño renal según el estadio de la enfermedad. Esto es posible haciendo una búsqueda activa de casos, realizando pruebas de cribado y haciendo intervenciones interdisciplinarias que incluyan educación de la población y del personal en salud, para así promover el autocuidado y realizar un seguimiento que impacte de forma positiva en la calidad de vida de los pacientes, en los desenlaces de la enfermedad y en la economía de los países en vía de desarrollo, los cuales deben optimizar sus limitados recursos e invertir en los procesos que generen mayor costo-efectividad.

Palabras clave: enfermedad renal crónica, prevención, salud pública, Colombia.

$\square$ Correspondencia: Cra. 27 No. 10-02, edificio 11, Pereira, Risaralda, Colombia. jzakzukperez@gmail.com 


\section{Ideal nefroprevention model for Colombia}

\section{Abstract}

Chronic kidney disease (CKD) is a highly prevalent entity in developing countries, affecting a large part of the Colombian population, which affects the quality of life, longevity and health costs. It has a progressive and irreversible character, so that intervening in early stages, turns out to be a highly costeffective measure, from this, the concept of nephro-prevention arises, in which taking into account the levels of primary, secondary and tertiary prevention, it is possible to obtain an early identification, interruption or slowing down of progression, and timely and comprehensive management of kidney damage according to the stage of their disease; taking into account an active search for cases, carrying out screening tests and an interdisciplinary intervention that includes education of the population and health personnel, in order to generate self-care and follow-up that positively impacts quality of life, outcomes and finally the economy of developing countries, which those who, with limited resources, must optimize and invest in the processes that generate greater cost-effectiveness.

Keywords: chronic renal insufficiency, prevention, public health, Colombia.

\section{Introducción}

La enfermedad renal crónica (ERC) se considera hoy día como un problema de salud pública que va en aumento [1]. Su importancia radica en la carga de la enfermedad, en su carácter progresivo e irreversible, en su letalidad y en los costos que acarrea, pues esta afecta aún más a los países en desventaja en donde gran parte de la población desconoce su condición y por tanto no ha sido tratada; esta situación lleva a mayores tasas de pacientes con ERC diagnosticados en estadios avanzados [2,3]. Lo anterior, sumado a problemas propios de los países en vía de desarrollo, como la insuficiencia del número de nefrólogos para cubrir la gran cantidad de pacientes y la falta de acceso a los medicamentos y terapia de reemplazo renal (TRR), hacen que las consecuencias de la ERC sean más graves [4,5].

La ERC está presente en 1 de cada 10 adultos a nivel mundial, y se proyecta que para 2040 sea la quinta causa más común de años perdidos [6]. Esta alta prevalencia también se puede ver en Latinoamérica, en donde está dada no solo por las enfermedades más prevalentes globalmente como la diabetes y la hipertensión arterial (HTA), sino también por las enfermedades infecciosas y una actividad nefrológica (asistencial y educativa) muy poco desarrollada, sobre todo en países con mayor porcentaje de habitantes mayores de 65 años como Argentina, Brasil, Chile y Uruguay.

De igual forma, se sabe que entre 1991 y 2013 la prevalencia de enfermedad renal en estadio terminal en pacientes sometidos a TRR se incrementó de 119 pacientes por millón de 
población (pmp) a 660 pmp [4]. Esto podría estar relacionado con el nivel socioeconómico, ya que se ha evidenciado que la pobreza incrementa el riesgo de presentar enfermedades que predisponen al desarrollo o a la progresión de la ERC y empeora los desenlaces en aquellos pacientes que ya la poseen debido a que la prosperidad incrementa el acceso a TRR. También se ha establecido que las personas en el cuartil socioeconómico más bajo tienen $60 \%$ más riesgo de ERC progresiva que aquellas que se encuentran en el cuartil más alto [7].

En Colombia, según la Cuenta de Alto Costo, entre julio de 2017 y junio de 2018 fueron reportados 1.479 .733 personas con ERC, evidenciando una prevalencia ajustada por edad de 2,9 casos por cada 100 habitantes y un aumento de 295.260 pacientes en este

\section{Un enfoque claro en nefroprevención para detener el avance de la enfermedad y reducir la mortalidad} periodo [8]. A pesar de esta prevalencia tan elevada, la mayoría de los pacientes no tiene un diagnostico establecido: solo el $6 \%$ de la población general y el $10 \%$ de la población de alto riesgo tienen claro su estatus de ERC [9], lo cual lleva a que pacientes diabéticos e hipertensos tengan un diagnóstico tardío [10].

Al no presentar síntomas sino hasta que el $90 \%$ de su función renal se encuentre deteriorada, los pacientes difícilmente cambian sus estilos de vida y las complicaciones, costos y mortalidad aumentan [11-13]; esto se puede ver reflejado en la esperanza media de vida a los 65 años, que en Latinoamérica es de 9 años, mientras que en Europa es de 14 años [14].

Debido a las limitaciones económicas que presentan los países en vía de desarrollo y las poblaciones desfavorecidas, es necesario que se establezca un enfoque claro en nefroprevención para detener el avance de la enfermedad y reducir la mortalidad, pues un diagnóstico temprano permite optimizar los recursos limitados a partir de métodos de cribado y del tratamiento de las comorbilidades, ya que se ha visto que las intervenciones son más efectivas si se realizan tempranamente $[15,16]$.

\section{Definición y clasificación de la ERC}

Para el año 2002, la National Kidney Foundation (NKF) publicó las guías K/DOQI (Kidney Disease Outcome Quality Initiative), en donde se estableció tanto la definición universal de la ERC como su evaluación y clasificación, siendo esto una apertura al reconocimiento de la importancia de esta patología durante la última década. De esta forma, la ERC se definió como un daño renal o una tasa de filtrado glomerular (TFG) $<60 \mathrm{~mL} / \mathrm{min} / 1,73 \mathrm{~m}^{2}$ presente 
durante al menos tres meses, considerando el daño renal como la presencia de anormalidades estructurales o funcionales del riñón que puedan provocar potencialmente un descenso de la TFG. Más adelante, en el año 2012, en las guías KDIGO (Kidney Disease: Improving Global Outcomes) se incorporó a la definición y clasificación la evaluación de las causas de la ERC y de la albuminuria, respectivamente, ya que estos factores pueden impactar en el pronóstico y la elección del tratamiento [17-19]. En la actualidad, la clasificación se realiza según la TFG (tabla 1).

Tabla 1: Clasificación por severidad de la enfermedad renal crónica según la guía KDIGO 2012.

\begin{tabular}{|c|c|c|}
\hline Estadio & Descripción & TFG $\mathrm{ml} / \mathrm{min} / \mathbf{1}, 73 \mathrm{~m}^{2}$ \\
\hline G1 & Daño renal con TFG normal o aumentada & $>90$ \\
\hline $\mathrm{G} 2$ & Daño renal con TFG media & $60-89$ \\
\hline G3a & Disminución moderada TFG & $45-59$ \\
\hline $\mathrm{G} 3 \mathrm{~b}$ & Disminución moderada a severa TFG & $30-44$ \\
\hline G4 & Disminución severa TFG & $15-29$ \\
\hline G5 & Falla renal & $<15$ (Diálisis) \\
\hline
\end{tabular}

Fuente: elaboración propia $[18,20]$.

\section{Factores de riesgo modificables y no modificables}

Los factores de riesgo para presentar ERC, más allá de tener una moción de ser resaltados, tienen la repercusión de suscitar un cambio; por consiguiente, se deben catalogar como aquellos que se pueden o no modificar.

Dentro de los factores modificables se encuentran la HTA y la diabetes mellitus. Esta última representa el 30-50\% de todos los casos de ERC y afecta a cerca del 6,4 \% de los adultos en todo el mundo, aunque se espera que este porcentaje aumente a $69 \%$ en países de altos ingresos y a $20 \%$ en países de bajos y medianos ingresos para 203020 . En este grupo también están la obesidad y el haber presentado una única vez daño renal agudo [21,22]. En países en vía de desarrollo se pueden observar, además, factores de tipo infeccioso, nefrotóxico o desconocido, exacerbados por el uso indiscriminado de analgésicos y la exposición al humo de cigarrillo, la cual genera un estado pro inflamatorio, estrés oxidativo, cambio glomeruloesclerosis y atrofia tubular $[23,24]$. 
Por otra parte, dentro del grupo de factores de riesgo no modificables se encuentra la historia familiar (cerca del $23 \%$ de los pacientes de diálisis incidentes tenían parientes cercanos con ERC [25]), el género (la ERC se presenta con mayor frecuencia en hombres [26]), la etnia, la edad (más de la mitad de los sujetos de edad avanzada examinados tenían ERC en etapas III-V según reporte de la guía K/DOQI) y la genética (el gen de apolipoproteina 1(APOL1) es mayormente recurrente en la etnia africada sub-sahariana [25, 27]).

Otros factores, modificables y no modificables, menos frecuentes serian la poliquistosis renal u otra anomalía estructural o congénita, ya sea renal o de la vía urinaria, como glomerulonefritis primaria y riñón solitario; la inadecuada hidratación con depleción recurrente de volumen, estrés por calor, exposición a pesticidas y metales pesados (se ha especulado que esta es la causa de la nefropatía mesoamericana) y, posiblemente, la dieta alta en proteínas en paciente de alto riesgo [28].

Conocer y detectar estos factores de riesgo aumenta la posibilidad de identificación temprana, lo que se traduce en el inicio oportuno del tratamiento para aquellos que son modificables y en el seguimiento adecuado para los que no se pueden modificar.

\section{Estrategia de nefroprevención}

La nefroprevención es una agrupación de preceptos destinados a evitar la aparición de daño renal primario en aquellos pacientes en quienes se han determinado factores de riesgo claros para su desarrollo, y, de no ser posible lo anterior, retardar el progreso de la ERC diagnosticada en pacientes con patologías metabólicas, cardiovasculares y/o nefrourológicas priorizando el control de los rangos de tensión arterial y la proteinuria [29]. De acuerdo a la definición de prevención según el Center of Disease Control and Prevention [30], estas actividades se caracterizan por su participación en los siguientes tres niveles:

1. Prevención primaria: se busca intervenir antes de que ocurran los efectos en la salud.

2. Prevención secundaria: se realiza cribado para identificar enfermedades en etapas tempranas, es decir, antes del inicio de signos o síntomas.

3. Prevención terciaria: se realiza manejo de la enfermedad posterior al diagnóstico para disminuir o parar su progresión.

En la Figura 1 se ilustra cada nivel de prevención con sus objetivos específicos, siendo estos los pilares para el desarrollo de estrategias de nefroprevención; ahora, estos se deben implementar desde la educación tanto para el personal de salud como para la población general, los grupos de autoayuda y las guías de manejo [2]. 


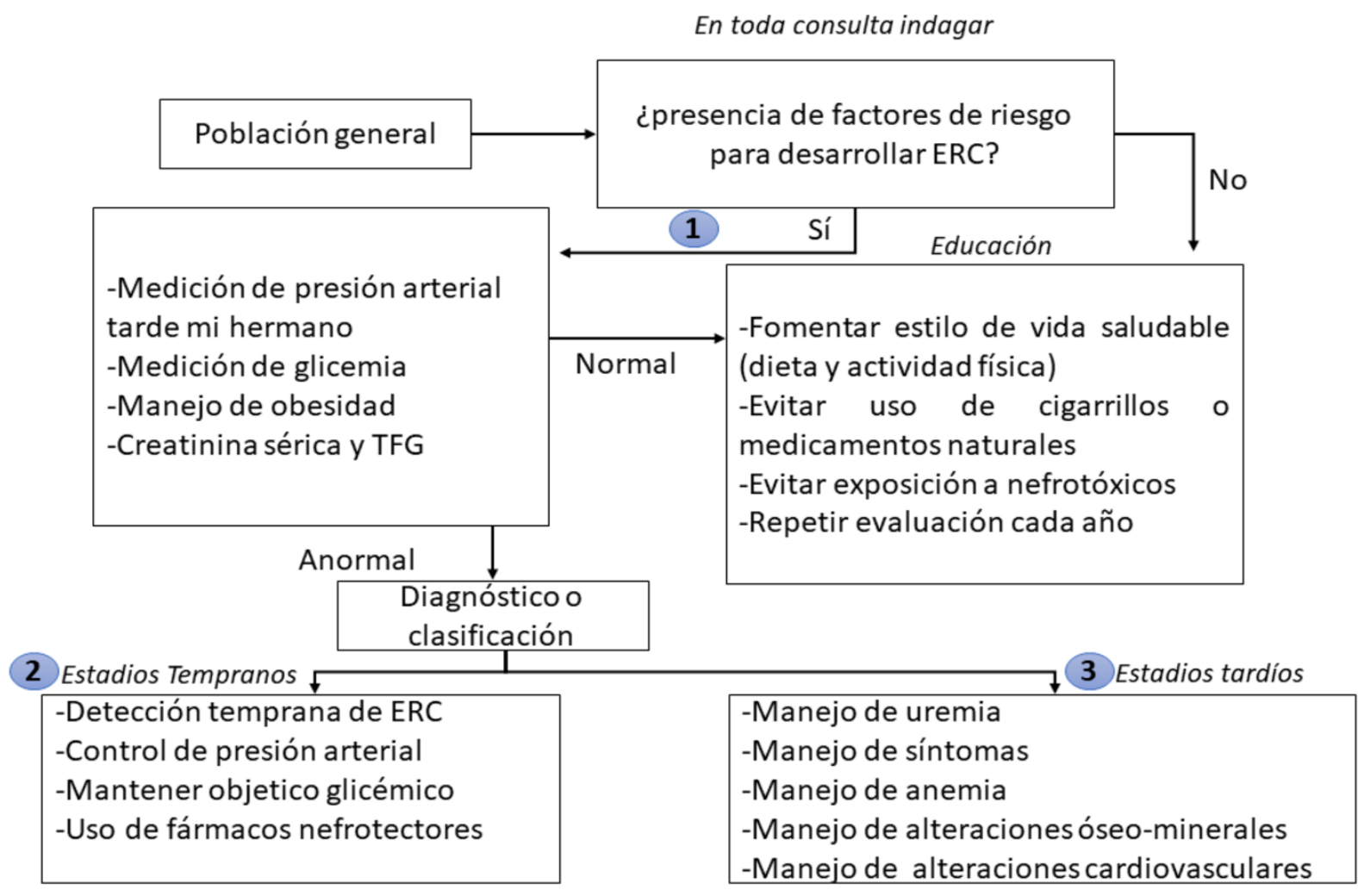

Figura 1: Modelo ideal de nefroprevención con niveles primario, secundario y terciario.

ERC: enfermedad renal crónica.

Fuente: elaboración propia.

Los objetivos de este modelo de nefroprevención son los siguientes:

1. Identificar de forma precoz al paciente con ERC entre la población general o entre quienes tienen factores de riesgo para su desarrollo.

2. Facilitar el abordaje inicial estableciendo un proceso sistematizado de derivación asistencial entre atención primaria y atención especializada.

3. Proporcionar información útil y fácil de entender a pacientes y cuidadores, creando consciencia acerca de la ERC, para que puedan ser partícipes de su proceso.

4. Integrar de forma eficaz las distintas opciones de manejo terapéutico (farmacológico, no farmacológico y educativo) para reducir el riesgo de progresión del daño renal y vascular asociado a la ERC.

5. Disminuir las barreras sanitarias propias de los países en desventaja.

6. Incrementar el proceso educativo dirigido al personal de salud presente en todos los niveles de atención con el fin de que este se encuentre capacitado en abordaje integral de pacientes con ERC. 


\section{Modelo ideal de nefroprevención en Colombia}

\section{Prevención primaria}

Para iniciar con la prevención primaria es necesario contar con una guía de cuidado del paciente que permita al personal de salud brindar una atención sistematizada, continua e integral bajo condiciones viables para evitar el deterioro y/o la rápida disminución de la función renal en pacientes no diagnosticados con ERC $[25,26]$. Además, se requieren espacios de educación para el personal de salud responsable de realizar el reconocimiento inicial, ya que se ha evidenciado que, por lo general, estos no tienen los conocimientos suficientes para abordar a esta población [31] y se ha demostrado que una educación adecuada puede incrementar sustancialmente las competencias de los clínicos para mejorar la preservación de la función renal en pacientes con ERC [32].

En este sentido, es necesario investigar acerca de posibles factores de riesgo para desarrollar ERC en la población general, para lo cual se debe indagar sobre la historia familiar de enfermedades y la probabilidad de portabilidad genética de las mismas, además de la introspección de patologías crónicas (¿cuáles son?, ¿qué son?, ¿cuáles son sus causas, sus factores de riesgo y de progresión?, ¿cómo se pueden mejorar?), y aspectos asociados como el síndrome metabólico y la alta ingesta calórica, que tienen igual importancia que la obesidad [33].

De igual forma, en este primer nivel de prevención es necesario promover estilos de vida saludable que incluyan actividad física y una dieta balanceada basada en vegetales, con menos carne, baja en sodio, con más carbohidratos complejos, alta en fibra y con menos grasas saturadas. En

Es necesario contar con una guía de cuidado del paciente que permita al personal de salud brindar una atención sistematizada pacientes monorrenos se sugiere, además, según un panel de expertos, que la ingesta proteica se limite a $1 \mathrm{~g} / \mathrm{kg}$ de peso por dia [34]. En los pacientes hipertensos es primordial optimizar los niveles de presión arterial y en los diabéticos, tener un control glicémico adecuado, lo cual ha demostrado ser efectivo para prevenir la nefropatía [35]. Adicionalmente, es importante alertar sobre los medicamentos nefrotóxicos, el hábito tabáquico y el cuidado de los pacientes en el uso de medios de contraste.

En este punto existe una barrera, que es la cobertura de salud en Colombia. A pesar de que esta ha aumentado y era de $95.07 \%$ a finales de 2020 [36], no es total debido a la falta de acceso 
en las zonas rurales y a la singularidad económica, por lo que se sugiere que estas personas sean abordadas primeramente en la zona rural donde habitan, para que mediante la educación y evaluación clínica puedan tener conocimiento de su riesgo o probabilidad de riesgo para ser atendidos posteriormente en ciudades que cuenten con instituciones que dispongan de atención multidisciplinaria e inicien inmediatamente con los cambios en su estilo de vida.

\section{Prevención secundaria}

Ya habiendo esclarecido quiénes son los pacientes con factores de riesgo o que ya cuentan con un deterioro de su función renal, se debe comenzar a aplicar la prevención secundaria iniciando con lo más básico y accesible: buen control de comorbilidades, optimización de la presión arterial y manejo de glicemia dentro de los rangos. Dada la naturaleza asintomática de la ERC, el cribado juega un rol importante en la detección temprana, ya que permite reconocer los individuos en quienes ya se inició el daño renal pero no han presentado signos o síntomas; por tanto, sería ideal realizarlo en toda la población, pero por temas de costo-efectividad la búsqueda de casos se realiza teniendo en cuenta factores de riesgo como edad mayor a 65 años, diabetes mellitus, HTA, historia familiar de ERC o exposición de drogas nefrotóxicas.

Dentro de los exámenes básicos a solicitar se encuentran la cuantificación de creatinina sérica, la estimación de la TFG, la determinación de marcadores de daño renal (particularmente albuminuria-proteinuria) y el análisis de sedimento urinario. Es importante mencionar que la detección de proteinuria en pacientes con HTA y diabetes ha demostrados ser costoefectiva [37] y que en casos especiales es necesario desarrollar otros estudios (usualmente en consulta con el nefrólogo), como imágenes, exámenes urinarios especiales y biopsia renal $[38,39]$.

La evidencia sugiere que la mayoría de los pacientes con ERC se encuentran en estadio inicial [20], por lo que es sumamente importante plantear estrategias para lograr identificarlos en este punto de la enfermedad y así poder generar un impacto real al contener o enlentecer la progresión de su deterioro renal [27,40]. Gran parte de estos pacientes pueden ser manejados en el primer nivel $[41,42]$, pero allí la evaluación debe ser sistematizada e incluir medidas de seguimiento que sean simples y fáciles de aplicar en ese entorno de atención [38,39], además se debe garantizar un manejo conjunto con el especialista.

De esta forma, referir al nefrólogo es esencial en el manejo de la ERC. Una revisión de Cochrane reportó que una referencia temprana (más de 1-6 meses antes del inicio de diálisis) se asoció a un $44 \%$ de reducción de la mortalidad a 5 años y acortamiento de la estancia hospitalaria [43], por lo que todo paciente diagnosticado debe ser remitido inmediatamente 
y, según su estadio, se debe establecer cada cuanto debe asistir a controles. Sin embargo, una de las barreras que enfrenta Latinoamérica es el déficit de nefrólogos, pues en Colombia, por ejemplo, se estima que solo hay 13,4 de estos especialistas pmp [4]. Entonces, un objetivo adicional del modelo ideal de nefroprevención sería aumentar el número de estos profesionales para poder abarcar la gran cantidad de pacientes con ERC que necesitan un seguimiento integral.

En cuanto a la terapia farmacológica, uno de los aspectos más importantes en la prevención secundaria es el uso de moduladores de la vía angiotensina-aldosterona como los Inhibidores de la enzima convertidora de angiotensina y los agonistas de los receptores de angiotensina II, los cuales reducen tanto la presión arterial sistémica como la intraglomerular mediante la apertura de las arteriolas eferentes glomerulares, extendiendo así la longevidad del resto de las nefronas [27].

Algunos hipoglucemiantes han demostrado un enlentecimiento de la progresión del daño renal, incluso en pacientes con ERC estadio III con HbA1c entre $7 \%$ y $8 \%$ [44]. Por su parte, los inhibidores del transportador sodio-glucosa tipo 2 (SGLT2i) y péptido similar a glucagón-1 pueden generar beneficios adicionales al control de la glicemia como mejorar los desenlaces renales y cardiovasculares. En el estudio EMPA-REG [45], la empaglifozina redujo en un $38 \%$ el riesgo de aparición o progresión de nefropatía y en un $44 \%$ la duplicación de creatinina sérica, comparado con placebo en pacientes con diabetes tipo 2 y alto riesgo cardiovascular.

De igual manera, se ha observado un posible beneficio de la canagliflozina en cuanto a la progresión de la albuminuria (HR: 0,73, IC95\%: 0,67-0,79) con una reducción sostenida del $40 \%$ en la TFG estimada, la necesidad de TRR o la muerte por causas renales (HR: 0,60, IC95\%: 0,47-0,77) [46]. También, se han evidenciado resultados similares con la dapagliflozina en cuanto a sus efectos nefroprotectores (disminución en los desenlaces renales) [47].

A pesar de contar con estas opciones farmacológicas, un número considerable de pacientes con ERC no tienen un control adecuado de sus factores de riesgo, y entre el $30 \%$ y $50 \%$ de los pacientes con HTA y diabetes no alcanzan sus objetivos terapéuticos [33], por lo que necesitan un enfoque integral y un compromiso personal para poder tener los resultados deseados.

\section{Prevención terciaria}

Por último, la prevención terciaria consiste en que, posterior a un diagnostico establecido según las guías KDIGO [18], se debe diseñar un plan de manejo según el estadio de ERC teniendo en cuenta la TFG, las necesidades individuales de cada paciente y sus factores de 
riesgo, adicional a los hábitos y la terapia farmacológica antes explicada. El éxito de este tipo de prevención, sobre todo en los pacientes con ERC en estadios tempranos, depende de la retroalimentación activa y la educación continua del personal de salud, la participación activa de los pacientes y la implementación de recursos financieros y administrativos enfocados al manejo de esta enfermedad tan prevalente [2].

En pacientes con estadios avanzados el manejo de la uremia y de las condiciones comórbidas relacionadas como la anemia, los trastornos óseos y minerales y la enfermedad cardiovascular es lo principal para que su longevidad pueda ser extendida [27]. Además, en estos pacientes la enfermedad cardiovascular es bastante alta, sobre todo si adicionalmente tienen diabetes o HTA [27]. Asimismo, a este punto están próximos a necesitar TRR en forma de diálisis o trasplante de riñón; sin embargo, en algunos con comorbilidades adicionales como cáncer metastásico se consideran las medidas paliativas.

Las intervenciones de nefroprevención que más generan impacto son aquellas que incluyen una participación autodidacta del paciente y supervisada por profesionales en salud. La educación enfocada en las necesidades individuales de cada paciente es muy importante, pues les permite conocer a fondo su enfermedad y los factores que la desencadenan o agravan, mejorar su confianza, disminuir síntomas y, por tanto, mejorar los desenlaces y su calidad de vida [48, 49]. El personal de salud, que debe estar entrenado para eso, tiene que interactuar de forma idónea con el paciente, realizar varias preguntas para detectar factores de riesgo reales, remover las barreras que se puedan presentar y promover jornadas de educación con los recursos que disponga cada comunidad [2].

Adicionalmente, los grupos de interacción y autoayuda son efectivos, pues se han evidenciado efectos positivos con su uso, ya sea en pacientes de riesgo (sin ERC) y con ERC temprana [2]. En dicho aspecto se promueve la participación activa de los pacientes, tanto en el autocuidado como en la comunicación de situaciones con las que pueden identificarse con otros pacientes, incrementando así su motivación para resolver y completar objetivos y abandonar hábitos tóxicos $[48,49]$.

Vale la pena mencionar que también se han probado nuevas estrategias, como por ejemplo aplicaciones o programas móviles por mensajes de texto, que permiten tener un seguimiento continuo y mejorar la actitud del paciente ante su patología, lo cual suscitaría una mejoría en el primer nivel de atención ya que permite acceder fácilmente a los pacientes y mantener contacto directo con ellos [50,51]. 


\section{Conclusiones}

Para finalizar, se puede concluir que la prevención de la ERC en Colombia constituye uno de los mayores problemas de salud pública, pues la culturización, la falta de inversión en salud y la carencia de conocimiento en promoción y prevención por parte de los trabajadores de la salud ocasionan falencias en materia de la detección precoz. De esta forma, se hace énfasis en que estos aspectos deben ser mejorados con estrategias multidisciplinarias bien enfocadas, con énfasis en la prevención primaria, secundaria y terciara, y teniendo en cuenta a la población susceptible que no cuenta con acceso a la atención sanitaria.

De igual forma, se debe individualizar de manera integral las necesidades de cada paciente y establecer una comunicación idónea entre el médico general y el nefrólogo, de tal modo que se utilice un modelo de atención sistematizado y bien desarrollado con el fin de reducir la incidencia de la ERC, desacelerar su rápida progresión e impactar directamente tanto en los costos de la enfermedad como en la calidad de vida del paciente.

\section{Consideraciones éticas}

Los autores declaran que los procedimientos seguidos se realizaron conforme a las normas éticas del comité de experimentación humana responsable y de acuerdo con lo establecido por la Asociación Médica Mundial en la Declaración de Helsinki; que han seguido los protocolos de su centro de trabajo sobre la publicación de datos de pacientes, y que han obtenido el consentimiento informado de los pacientes y/o sujetos referidos en el artículo.

\section{Contribución de los autores}

Todos los autores participaron activamente en la concepción y diseño del trabajo, la bús-

queda bibliográfica y la redacción, revisión crítica y aprobación de la versión final del manuscrito.

\section{Conflicto de intereses}

Ninguno declarado por los autores.

\section{Financiación}

Ninguna declarada por los autores. 


\section{Referencias}

[1] Acuña L, Sánchez P, Soler LA, Alvis LF. Enfermedad renal en Colombia: 19Prioridad para la gestión de riesgo. Rev Panam Salud Publica. 2016;40(1):16- 22. $\uparrow$ Ver página 2

[2] Cueto-Manzano AM, Martínez-Ramírez HR, Cortés-Sanabria L, Rojas-Campos E. The Role of Primary Health Care Professionals. In: García-García G, Agodoa LY, Norris KC, editors. Chronic Kidney Disease in Disadvantaged Populations. Elsevier Inc.; 2017. p. 329-335. http: //dx.doi.org/10.1016/B978-0-12-804311-0.00031-5. ^Ver página 2, 5, 10

[3] Garcia-Garcia G, Jha V. Chronic kidney disease in disadvantaged populations. Indian J

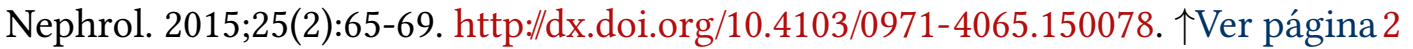

[4] Cusumano AM, Rosa-Diez GJ, Gonzalez-Bedat MC. Latin American Dialysis and Transplant Registry: Experience and contributions to end-stage renal disease epidemiology. World J Nephrol. 2016;5(5):389-97. http:/dx.doi.org/10.5527/wjn.v5.i5.389. 个Ver página 2, 3, 9

[5] Naicker S. End-stage renal disease in sub-Saharan Africa. Ethn Dis. 2009;19(1 Suppl 1): S1-13-15. $\uparrow$ Ver página 2

[6] Foreman KJ, Marquez N, Dolgert A, Fukutaki K, Fullman N, McGaughey M, et al. Forecasting life expectancy, years of life lost, and all-cause and cause-specific mortality for 250 causes of death: reference and alternative scenarios for 2016- 40 for 195 countries and territories. Lancet. 2018;392(10159):2052-90. http:/dx.doi.org/10.1016/S0140-6736(18)31694-5. 个Ver página 2

[7] Merkin SS, Diez-Roux AV, Coresh J, Fried LF, Jackson SA, Powe NR. Individual and neighborhood socioeconomic status and progressive chronic kidney disease in an elderly population: The Cardiovascular Health Study. Soc Sci Med. 2007;65(4):809-21. http:/dx.doi.org/ 10.1016/j.socscimed.2007.04.011. 个Ver página 3

[8] Colombia. Ministerio de Salud y Protección Social, Ministerio de Hacienda y Crédito Público. Cuenta de Alto Costo: Fondo Colombiano de Enfermedades de alto Costo. Situación de la Enfermedad Renal Crónica, Hipertensión Arterial y Diabetes Mellitus en Colombia 2017. Bogotá: Ministerio de Salud y Protección Social; 2017 [citado abril 11 2021]. Disponible en: https:/cuentadealtocosto.org/site/publicaciones/ situacion-de-la-enfermedad-renal-cronica-la-hipertension-arterial-y-la-diabetes-mellitus $\backslash$ -en-colombia-2017/?1584310319210. 个Ver página 3

[9] Palmer AJ, Valentine WJ, Chen R, Mehin N, Gabriel S, Bregman B, et al. A health economic analysis of screening and optimal treatment of nephropathy in patients with type 2 diabetes 
and hypertension in the USA. Nephrol Dial Transplant. 2008;23(4):1216-23. http:/dx.doi.org/ 10.1093/ndt/gfn082. 个Ver página 3

[10] Camargo JA, Vargas JG, D ’ Achiardi R, Echeverri JE. Factores de riesgo para la progresión de enfermedad renal crónica en pacientes con nefropatía diabética estadios 3 y 4 del Servicio de Nefrología del Hospital Militar Central de Bogotá. Rev. Colomb. Nefrol. 2014 [citado abril 11 2021];1(Suppl 1). Disponible en: https:/revistanefrologia.org/index.php/rcn/article/view/ 146. $\uparrow$ Ver página 3

[11] Jungers P. Late referral: loss of chance for the patient, loss of money for society. Nephrol Dial Transplant. 2002;17(3):371-375. http://x.doi.org/10.1093/ndt/17.3.371. $\uparrow$ Ver página 3

[12] Obrador GT, Pereira BJ. Early referral to the nephrologist and timely initiation of renal replacement therapy: a paradigm shift in the management of patients with chronic renal failure. Am J Kidney Dis. 1998;31(3):398-417. http:/dx.doi.org/10.1053/ajkd.1998.v31.pm9506677. 个Ver página 3

[13] Mathew T, Corso O. Review article: Early detection of chronic kidney disease in Australia: which way to go? Nephrology (Carlton). 2009;14(4):367-73. http://x.doi.org/10.1111/ j.1440-1797.2009.01113.x. $\uparrow$ Ver página 3

[14] Luxardo R, Kramer A, Gonzalez-Bedat MC, Massy ZA, Jager KJ, Rosa-Diez G, et al. The epidemiology of renal replacement therapy in two different parts of the world: the Latin American Dialysis and Transplant Registry versus the European Renal AssociationEuropean Dialysis and Transplant Association Registry. Rev Panam Salud Publica. 2018;42:e87. http:/dx.doi.org/10.26633/RPSP.2018.87. $\uparrow$ Ver página 3

[15] Cueto-Manzano AM, Martínez-Ramírez HR, Cortés-Sanabria L. Management of chronic kidney multidisciplinary disease: approach. Primary Clin health-care Nephrol. setting, selfcare 2010;74(Suppl and 1):99-104. http:/dx.doi.org/10.5414/cnp74s099. $\uparrow$ Ver página 3

[16] Cueto-Manzano AM, Martínez-Ramírez HR, Cortés-Sanabria L. Comparison of primary health-care models in the management of chronic kidney disease. Kidney Int Suppl.

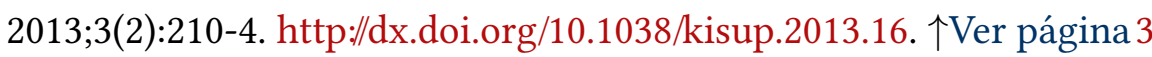

[17] National Kidney Foundation. Clinical practice guidelines for chronic kidney disease: evaluation, classification, and stratification. Am J Kidney Dis. 2002;39 (2 Suppl 1):S1-266. $\uparrow$ Ver página 4

[18] Idney Disease: Improving Global Outcomes (KDIGO) CKD Work Group. KDIGO 2012 Clinical Practice Guideline for the Evaluation and Management of Chronic Kidney Disease. Kidney Int Suppl. 2013;3(1):1-150. doi: http:/dx.doi.org/10.1038/kisup.2012.76 个Ver página 4, 9 
[19] Grupo de trabajo de la Guía de Práctica Clínica sobre la Detección y el Manejo de la Enfermedad Renal Crónica. Guía de Práctica Clínica sobre la Detección y el Manejo de la Enfermedad Renal Crónica. Madrid: Ministerio de Sanidad Servicios Sociales e Igualdad; 2016 [citado abril 12 2020]. Disponible en: https:/portal.guiasalud.es/wp-content/uploads/

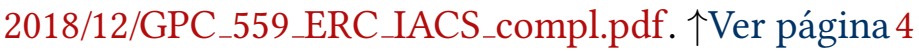

[20] Webster AC, Nagler E V., Morton RL, Masson P. Chronic Kidney Disease. Lancet. 2017;389(10075):1238-52. http:/dx.doi.org/10.1016/S0140-6736(16)32064-5. 个Ver página 4, 8

[21] Chang A, Kramer H. CKD progression: a risky business. Nephrol Dial Transplant.

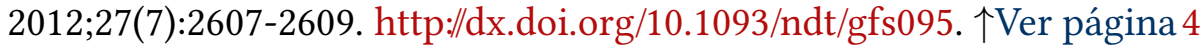

[22] Goldstein SL, Devarajan P. Acute kidney injury in childhood: should we be worried about progression to CKD? Pediatr Nephrol. 2011;26(4):509-522. http://x.doi.org/10.1007/ s00467-010-1653-4. $\uparrow$ Ver página 4

[23] Mirrakhimov AE. Obstructive sleep apnea and kidney disease: ¿is there any direct link?

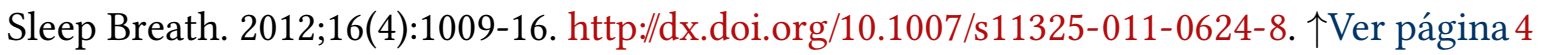

[24] Hsiao LL. Raising awareness, screening and prevention of chronic kidney disease: It takes more than a village. Nephrology (Carlton). 2018;23(Suppl 4):107-11. http://x.doi.org/10.1111/ nep.13459. $\uparrow$ Ver página 4

[25] Song EY, McClellan WM, McClellan A, Gadi R, Hadley AC, Krisher J, et al. Effect of community characteristics on familial clustering of end-stage renal disease. Am J Nephrol.

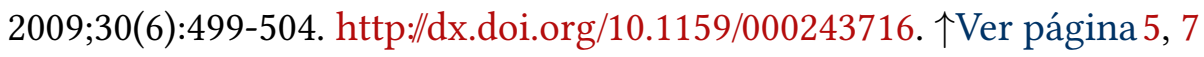

[26] Iseki K. Factors influencing the development of end-stage renal disease. Clin Exp Nephrol. 2005;9(1):5-14. http://x.doi.org/10.1007/s10157-005-0341-3. 个Ver página 5, 7

[27] Li PK, Garcia-Garcia G, Lui SF, Andreoli S, Fung WW, Hradsky A, et al. Kidney Health for Everyone Everywhere: From Prevention to Detection and Equitable Access to care. Clin Nephrol. 2020;93(3):111-22. http://dx.doi.org/10.14740/wjnu402. $\uparrow$ Ver página 5, 8, 9, 10

[28] Kalantar-Zadeh K, Fouque D. Nutritional Management of Chronic Kidney Disease. N Engl

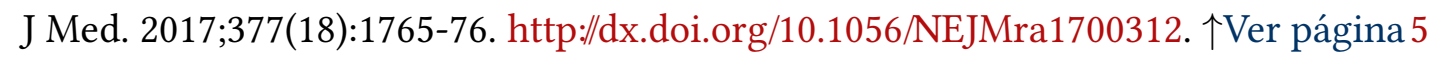

[29] Caggiani M, Halty M. Conceptos de nefroprevención. Arch pediatr Urug. 2009;80(3):2159. $\uparrow$ Ver página 5

[30] Centers for Disease Control and Prevention (CDC). Picture of America: Kindey Chronic Disease. Atlanta: CDC; 2019 [citado april 7 2020]. Disponible en: https:/www.cdc.gov/ pictureofamerica/pdfs/Picture_of_America_Chronic_Kidney_Disease.pdf. $\uparrow$ Ver página 5 
[31] Martínez-Ramírez HR, Cortés-Sanabria L, Rojas-Campos E, Barragán G, Alfaro G, Hernández $\mathrm{M}$, et al. How frequently the clinical practice recommendations for nephropathy are achieved in patients with type 2 diabetes mellitus in a primary health-care setting?. Rev Invest Clin. 2008;60(3):217-26. $\uparrow$ Ver página 7

[32] Cortes-Sanabria L, Cabrera-Pivaral CE, Cueto-Manzano AM, Rojas-Campos E, Barragán G, Hernández-Anaya M, et al. Improving care of patients with diabetes and CKD: a pilot study for a cluster-randomized trial. Am J Kidney Dis. 2008;51(5):777-88. http:/dx.doi.org/ 10.1053/j.ajkd.2007.12.039. $\uparrow$ Ver página 7

[33] Verhave JC, Troyanov S, Mongeau F, Fradette L, Bouchard J, Awadalla P, et al. Prevalence, awareness, and management of $\mathrm{CKD}$ and cardiovascular risk factors in publicly funded health care. Clin J Am Soc Nephrol. 2014;9(4):713-9. http:/dx.doi.org/10.2215/CJN.06550613. 个Ver página 7, 9

[34] Tantisattamo E, Dafoe DC, Reddy UG, Ichii H, Rhee CM, Streja E, et al. Current Management of Patients With Acquired Solitary Kidney. Kidney Int Rep. 2019;4(9):1205-18.

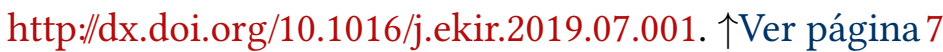

[35] Ene-Iordache B, Perico N, Bikbov B, Carminati S, Remuzzi A, Perna A, et al. Chronic kidney disease and cardiovascular risk in six regions of the world (ISN- KDDC): a cross-sectional study. Lancet Glob Heal. 2016;4(5):e307-19. http://x.doi.org/10.1016/ S2214-109X(16)00071-1. 个Ver página 7

[36] Colombia. Ministerio de Salud y Protección Social (MinSalud). Colombia sigue avanzando en la cobertura universal en salud. Bogotá D.C.: MinSalud; 2020 [citado mayo 15]. Disponible en: https:/www.minsalud.gov.co/Paginas/ Colombia-sigue-avanzando-en-la-cobertura-universal-en-salud-.aspx\#: :text=\%E2\% 80\%8B\%2D\%20Ante\%20la\%20OMS\%2C\%20el,la\%20regi\%C3\%B3n\%20de\%20las\%20am\%C3\% A9ricas. $\uparrow$ Ver página 7

[37] Komenda P, Ferguson TW, Macdonald K, Rigatto C, Koolage C, Sood MM, et al. Cost-effectiveness of primary screening for CKD: a systematic review. Am J Kidney Dis.

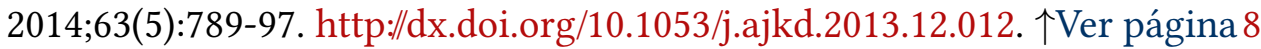

[38] Harris D, Thomas M, Johnson D, Nicholls K, Gillin A. The CARI guidelines. Prevention of progression of kidney disease. Nephrology (Carlton). 2006;11(Suppl 1):S2-197. https://doi. org/10.1111/j.1440-1797.2006.00505.x. 个Ver página 8

[39] Cueto-Manzano AM, Cortes-Sanabria L, Martínez-Ramírez HR, Rojas-Campos E. Enfermedad Renal Crónica Temprana. Prevención, Diagnóstico y Tratamiento. México D.F.: Editorial Médica Panamericana; 2013. $\uparrow$ Ver página 8 
[40] Feehally J, Brusselmans A, Finkelstein FO, Harden P, Harris D, Manuzi G, et al. Improving global health: measuring the success of capacity building outreach programs: a view from the International Society of Nephrology. Kidney Int Suppl (2011). 2016;6(2):42-51. http:/dx.

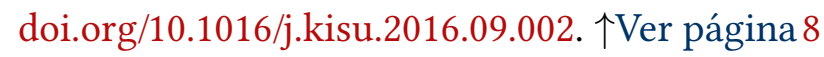

[41] Jones CA, Francis ME, Eberhardt MS, Chavers B, Coresh J, Engelgau M, et al. Microalbuminuria in the US population: third National Health and Nutrition Examination Survey. Am J Kidney Dis. 2002;39(3):445-459. http:/dx.doi.org/10.1053/ajkd.2002.31388. 个Ver página 8

[42] Amato D, Alvarez-Aguilar C, Castaneda-Limones R, Rodriguez E, Avila-Diaz M, Arreola $\mathrm{F}$, et al. Prevalence of chronic kidney disease in an urban Mexican population. Kidney Int

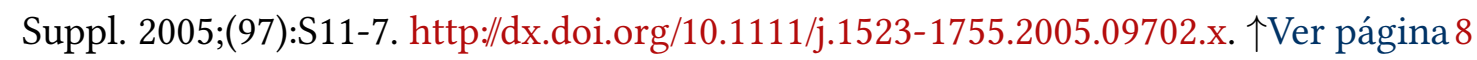

[43] Smart NA, Dieberg G, Ladhani M, Titus T. Early referral to specialist nephrology services for preventing the progression to end-stage kidney disease. Cochrane database Syst Rev. 2014;(6):CD007333. http://dx.doi.org/10.1002/14651858.CD007333.pub2. $\uparrow$ Ver página 8

[44] Lopera-Vargas JM, Rico-Fontalvo JE, Melgarejo E, Castillo-Barrios GE, Ramírez-Rincón A, Gómez AM, et al. Efecto de terapias farmacológicas para el control glicémico en pacientes con Diabetes Mellitus tipo 2 en los desenlaces vasculares. Rev Colomb Nefrol. 2020;7(1):4459. http:/dx.doi.org/10.22265/acnef.7.1.372. $\uparrow$ Ver página 9

[45] Zinman B, Wanner C, Lachin JM, Fitchett D, Bluhmki E, Hantel S, et al. Empagliflozin, Cardiovascular Outcomes, and Mortality in Type 2 Diabetes. N Engl J Med.

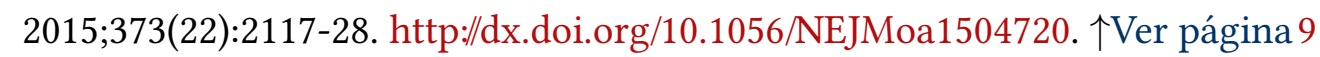

[46] Arocha-Rodulfo JI, Amair P, Marantes D, Navas-Blanco T. El papel del riñón en la homeostasis de la glucosa y drogas de acción renal en el tratamiento de la diabetes mellitus

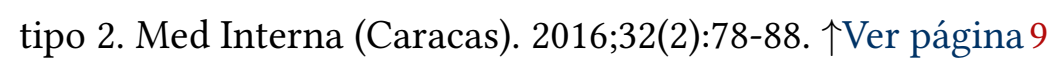

[47] Neal B, Perkovic V, Mahaffey KW, de Zeeuw D, Fulcher G, Erondu N, et al. Canagliflozin and Cardiovascular and Renal Events in Type 2 Diabetes. N Engl J Med. 2017;377(7):644-57. http:/dx.doi.org/10.1056/NEJMoa1611925. $\uparrow$ Ver página 9

[48] Coulter A, Ellins J. Effectiveness of strategies for informing, educating, and involving patients. BMJ. 2007;335(7609):24-7. http:/dx.doi.org/10.1136/bmj.39246.581169.80. 个Ver página 10

[49] Organización Mundial de la Salud (OMS). Informe sobre la salud en el mundo 2008. La atención primaria de salud, más necesaria que nunca. Ginebra: OMS; 2008 [citad0 abril 8 2020]. Disponible en: https:/www.who.int/whr/2008/es/. $\uparrow$ Ver página 10 
[50] Cueto-Manzano AM, Gallardo-Rincón H, Martínez-Ramírez HR, Cortés- Sanabria L, Rojas-Campos E, Tapia-Conyer R, et al. A pilot study of a mobile phone application to improve lifestyle and adherence of patients with kidney disease. J Telemed Telecare.

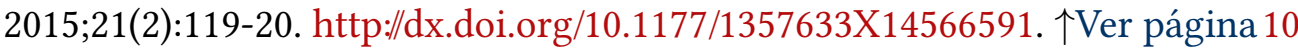

[51] Diamantidis CJ, Ginsberg JS, Yoffe M, Lucas L2, Prakash D3, Aggarwal S, et al. Remote Usability Testing and Satisfaction with a Mobile Health Medication Inquiry System in CKD. Clin J Am Soc Nephrol. 2015;10(8):1364-70. http:/dx.doi.org/10.2215/CJN.12591214. 个Ver página 10 